\title{
VALIDACIÓN DEL INVENTARIO DE PERCEPCIONES Y CREENCIAS ANTE EL DOLOR EN ARGENTINOS CON CEFALEAS
}

\author{
Luciana Sofía Moretti ${ }^{1}$ \\ Leonardo Adrián Medrano \\ Pontificia Universidad Católica Madre y Maestra, Republica Dominicana
}

\begin{abstract}
RESUMEN
El objetivo del presente es validar el Inventario de Percepciones y Creencias ante el Dolor (IPCD). Mediante un muestreo intencional se seleccionaron 382 personas de Córdoba (Argentina) que en los últimos seis meses habían sufrido de cefaleas. Se realizaron estudios para evaluar las propiedades psicométricas del instrumento analizando la estructura interna, consistencia interna, y la validez externa del cuestionario. Para ello, se llevó a cabo un análisis factorial exploratorio que reveló una estructura de 3 dimensiones: Autoinculpación $(\alpha=, 83)$; Dolor como un Misterio $(\alpha=, 79)$ y Cronicidad del Dolor $(\alpha=, 80)$, teóricamente coherentes con las dimensiones planteadas en investigaciones previas. Sumado a ello, se obtuvieron evidencias de validez satisfactorias al considerar la intensidad del dolor como variable criterio. A partir de los resultados obtenidos puede afirmarse que el IPCD permite examinar de manera válida y confiable percepciones y creencias asociadas al dolor, permitiendo contar con una herramienta adecuada tanto para fines clínicos como de investigación.
\end{abstract}

\section{Palabras clave}

Creencias; Validación; Cefaleas; Dolor crónico.

\begin{abstract}
The objective of this paper is to validate the Inventory of Perceptions and Beliefs in the Face of Pain (IPCD). An intentional survey selected 382 people from Córdoba, Argentina, who had suffered from headaches in the last six months. Studies were conducted to evaluate the psychometric properties of the instrument by analyzing the internal structure, internal consistency, and external validity of the questionnaire. To this end, an exploratory factorial analysis was carried out, which revealed a 3-dimensional structure: Self-incrimination $(\alpha=, 83)$; Pain as a Mystery ( $\alpha$ $=, 79)$ and Chronicity of Pain $(\alpha=, 80)$, theoretically coherent with the dimensions proposed in previous research. In addition, evidence of satisfactory validity was obtained when considering pain intensity as a criterion variable. Based on the results obtained, it can be stated that the IPCD makes it possible to validly and reliably examine perceptions and beliefs associated with pain, thus providing an adequate tool for both clinical and research purposes.
\end{abstract}

\section{Keywords}

Beliefs; Psychometric validation; Headaches; Chronic pain.

\footnotetext{
${ }^{1}$ Correspondence about this article should be addressed to Luciana Sofía Moretti Email: 1smoretti@gmail.com
} 


\section{VALIDATION OF THE INVENTORY OF PERCEPTIONS AND BELIEFS BEFORE THE PAIN IN ARGENTINOS WITH CEFALEAS}

El estudio de los factores cognitivos en pacientes con dolor crónico se reconoce como un componente importante, y en los últimos años es un área que cada vez recibe cada vez mayor atención (Jackson, Wang \& Fan, 2014). En las últimas décadas se ha empezado a investigar el papel de estos factores cognitivos en la adaptación al dolor crónico, así como su influencia en la percepción final del dolor y en las estrategias de afrontamiento (Moretti, 2010).

En esta misma línea, las creencias han sido definidas como configuraciones cognitivas personalmente formadas o culturalmente compartidas (Fergus, 2013); son nociones preexistentes acerca de la naturaleza de la realidad, la cual moldea la percepción de las personas y de su ambiente configurando un significado (García-Camargo \& Acuña, 2016). En el ámbito específico del dolor crónico, las creencias son aquellas cogniciones (pensamientos) que las personas tienen en relación con su problema de dolor (Pons, Shipton \& Mulder, 2012). Asimismo, sirven para obtener conocimiento y comprensión de los eventos que sucedieron, suceden o sucederán (Stroud, Thorn, Jensen \& Boothby, 2000). De igual forma, las creencias de dolor son conceptualizaciones propias de cada persona sobre qué es el dolor y qué significa para ellas, y pueden ser entendidas como un subconjunto de un sistema de creencias el cual representa una comprensión personal de la experiencia de dolor (Pons et al., 2012). De acuerdo con Jackson et al. (2014), dependiendo de la persona y de la situación en la que se encuentre, las creencias primarias respecto del dolor influirán en las estrategias de afrontamiento que desplegará la persona.

Para DeGood y Shutty (1992) existen tres tipos de creencias sobre el dolor, ellas son: 1) suposiciones filosóficas básicas sobre la naturaleza de uno mismo y el mundo, 2) creencias generalizadas y estables y, por último, 3) creencias relacionadas con el contexto del dolor y su tratamiento. Con respecto a las primeras, este tipo de creencias engloban suposiciones sobre valores como la justicia, el sufrimiento, la responsabilidad. Por ejemplo, cuando una persona cree que la vida debería estar libre de dolor, este valor puede intensificar los sentimientos de sufrimiento asociados con el dolor (Ho \& Johnson, 2013). En relación a las segundas, existiría una tendencia a utilizar ciertas cogniciones que incidirían en el uso de distintas habilidades de afrontamiento: Con respecto al locus de control, las personas con fuertes creencias internas informan menor dolor y realizan menos conductas de dolor que aquellos con creencias internas débiles. Por otro lado, el estilo atribucional refiere a que el tipo de inferencia causal que realiza la persona sobre su dolor, incide sobre la intensidad percibida; y las expectativas de autoeficacia, representan una autovaloración del individuo sobre el control potencial que tiene en una situación. Por último, dentro de las creencias relacionadas con el contexto del dolor y su tratamiento están las creencias sobre la temporalidad (estabilidad del dolor), a partir de las cuales las personas que piensan que su dolor persistirá tienden a utilizar estrategias pasivas. Asimismo, se encuentran las creencias acerca del origen del dolor, a partir de las cuales los individuos que piensan que su dolor es un misterio, evalúan negativamente sus habilidades para controlarlo o disminuirlo. Por último, las creencias acerca del diagnóstico y/o tratamiento del dolor son las que determinan la adherencia a los tratamientos médicos. Es preciso destacar que existen otro tipo de creencias que estarían implicadas en las creencias de dolor, se tratan de las creencias de indefensión. Éstas son determinantes en la percepción que poseen los individuos acerca del tratamiento y son consideradas como un estado psicológico que se produce cuando los acontecimientos a los que se enfrentan son incontrolables e impredecibles (Moretti, 2010). En este sentido, diversos estudios revelan que las creencias de indefensión de las personas ante el dolor, se relacionan de forma positiva con la intensidad del dolor que perciben, malestar emocional y conductas de dolor (Ho \& Johnson, 2013; Pons et al., 2012).

Las creencias relacionadas al dolor se desarrollan como resultado de experiencias de aprendizaje previas y del contexto cultural de los sujetos (Turk \& Okifuji, 2002). Por lo que, según las experiencias que el individuo haya tenido con el dolor o la observación de personas cercanas que lo padezcan o lo hayan padecido así como también por las informaciones provenientes de los agentes de salud y sistemas 
de comunicación (Ho \& Johnson, 2013), el individuo aprende que el dolor tiene diferentes significados. Asimismo, dependiendo de la observación de los modos que tienen otras personas para responder ante el dolor en la cultura en la que se haya inserto el paciente, puede aprender a interpretar y/o a evaluar el dolor de diferentes maneras (Sharp, 2001). Más aun, hallazgos recientes apuntan hacia la existencia de procesos de autorregulación entre los pacientes con dolor crónico, en cuya adquisición y desarrollo parecen tener mayor peso los factores psicosociales que la propia experiencia. En otras palabras, la experiencia previa constituiría un factor relevante en la intensidad percibida del dolor, pero no parece tener la entidad suficiente para conformar distintos sistemas de creencias sobre el dolor crónico en función del padecimiento de distintos trastornos de esta naturaleza (Bylinka \& Oniszczenko, 2016; Ho \& Johnson, 2013).

Por otra parte, diversas investigaciones afirman que las percepciones y creencias asociadas al dolor, pueden influir en su afrontamiento y en la sensación de intensidad. Por tanto, las creencias desadaptativas ejercen una influencia desfavorable en el afrontamiento del dolor crónico reduciendo su eficacia (Vanhaudenhuyse et al., 2018). Específicamente, las creencias acerca de la experiencia de dolor con valor amenazante predicen el empleo de estrategias de afrontamiento más pasivas y centradas en la emoción, las personas exhiben mayores reacciones emocionales negativas y se observa una ejecución deficiente de las estrategias de afrontamiento; mientras que aquellas personas en las que las creencias se asocian a la valoración de desafío predicen el despliegue de estrategias de afrontamiento más activas, centradas en el problema, exhiben menor estrés subjetivo y se observan mejoras en la puesta en marcha de estrategias de afrontamiento (Jackson et al., 2014; Vanhaudenhuyse et al., 2018).

Los resultados obtenidos de las investigaciones realizadas en torno a las creencias acerca de los recursos para afrontar el dolor, muestran que éstas se relacionan de forma negativa con la intensidad del dolor percibido por el paciente. Es por esto que, diversos estudios afirman que las personas que creen tener recursos utilizan más autoafirmaciones de afrontamiento, mientras que los que tienen creencias de indefensión son más catastrofistas (Pons et al., 2012). En un estudio llevado a cabo por Williams y Keefe (1991; en Camacho Martel \& Anarte Ortiz, 2001) se concluyó que los individuos que se caracterizaban por tener creencias sobre la temporalidad del dolor y lo conceptualizaban como un misterio, fueron más catastrofistas, menos propensos a usar estrategias de afrontamiento cognitivas, y puntuaron menos en los índices de efectividad sobre las estrategias de afrontamiento que usaban para controlar y disminuir su dolor, en comparación con aquellos pacientes que comprendían la naturaleza del mismo. Por último, es importante remarcar que las creencias vinculadas al dolor crónico están asociadas con la intensidad de dolor reportado, el estado de ánimo y la incapacidad relacionada al dolor (Ho \& Johnson, 2013; Jackson et al., 2014; Vanhaudenhuyse et al., 2018).

Dada la relevancia que evidencian las creencias y percepciones acerca del dolor, resulta importante contar con instrumentos que permitan la medición de estos constructos. El Inventario de Percepciones y Creencias ante el Dolor (PBPI) de Williams y Thorn (1989) ha sido elaborado para estos fines. Originalmente, los autores del inventario propusieron tres dimensiones con el objeto de medir las creencias hacia el dolor: (a) Autoinculpación, (b) Percepción del dolor como un misterio, y (c) Creencias acerca de la duración del dolor. Desde su aparición, diversas investigaciones han dado cuenta de la confiablidad y validez de dicho instrumento (Condello, Piano, Dadam, Pinessi \& Lantéri-Minet, 2014; Dany, Rousse, Carayon, Blois \& Apostolidis, 2009; Goli, Yanchuk \& Torkaman, 2015; Gonzales, Soler \& Ferrer, 2002; Herda, Siegeris \& Basler, 1994; Mikail, D’Eon \& De Gagné, 1995; Monticone et al., 2013; Morley \& Wilkinson, 1995; Wong, Williams, Mak \& Fielding, 2011). Así, Herda et al. (1994) aplicaron el inventario a una muestra alemana de 193 pacientes con dolor. Los autores verificaron una estructura factorial de cuatro factores, denominados Constancia, Aceptación, Autoinculpación, y Dolor como un Misterio. Los índices de consistencia interna fueron aceptables para las dimensiones de Constancia, Aceptación, y Autoinculpación, presentando valores alfa de Cronbach entre .72 y .84; mientras que para la dimensión Dolor como un Misterio los valores de consistencia interna fueron bajos $(\alpha=.64)$. Por último, 
las cargas factoriales evidenciaron dos ítems complejos, con cargas considerables en más de un factor (ítems 5 y 2 ).

En uno de los estudios en el que se aplicó un análisis factorial confirmatorio de la escala, Mikail et al. (1995) analizaron el ajuste de un modelo de cuatro factores en una muestra de sujetos con dolor crónico. Los cuatro factores que integraron el modelo fueron: Cronicidad; Autoinculpación; Misterio; y Constancia del Dolor. Se obtuvieron índices de ajustes adecuados para el modelo propuesto $\left(\mathrm{CFI}=.90 ; \mathrm{R}^{2}=.997\right)$. Cabe mencionar que en el modelo se permitió solamente la covariación entre las variables latentes Cronicidad y Autoinculpación $(r=-, 20 ; p<, 001) ; y$ Cronicidad y Constancia del Dolor $(r=, 52 ; \mathrm{p}<, 001)$. Finalmente, se observaron índices de consistencia interna adecuada en la mayoría de las dimensiones (valores alfa entre .70 y .76), salvo en el factor Misterio $(\alpha=.63)$.

Por su parte, Wong et al. (2011) analizaron el instrumento en una muestra China de 218 pacientes con dolor musculoesquelético crónico. En este caso se aplicó un Análisis Factorial Confirmatorio, mediante el cual los autores verificaron el ajuste de cuatro modelos. Mediante estos modelos se intentó corroborar la validez factorial analizando separadamente las siguientes dimensiones: Misterio (i.e. modelo 1); Permanencia (i.e. modelo 2); Constancia (i.e. modelo 3); y Autoinculpación (i.e. modelo 4). Los índices de ajustes hallados para todos los modelos fueron adecuados (CFI y NFI $\geq .90$ ), y las cargas de los ítems en sus correspondientes factores fueron estadísticamente significativas $(\mathrm{p}<.05)$. Sin embargo, los coeficientes de consistencia interna verificados para las dimensiones fueron en su mayoría bajos (Misterio $\alpha=.65$; Permanencia $\alpha=$ .76; Constancia $\alpha=.60$; Autoinculpación $\alpha=.63$ ).

Dany, et al. (2009), adaptaron y validaron una versión francesa del PBPI en una muestra de 247 pacientes con dolor crónico. Los autores encontraron una estructura factorial de cuatro factores que explicaba el 57.7\% de la varianza en los ítems: Constancia, Permanencia, Culpabilidad, y Misterio. Al igual que en Herda et al. (1994), los ítems dos y cinco presentaron cargas factoriales considerables en más de un factor. Para todas las dimensiones se encontraron índices de confiabilidad adecuados (valores $\alpha \geq .70$ ), salvo para la dimensión Culpabilidad $(\alpha=$ $.63)$.

En la adaptación inglesa del instrumento, Morley y Wilkinson (1995) verificaron también una estructura factorial de cuatro dimensiones que explicaban el 60.3\% de la varianza. Al forzar la estructura factorial original de 3 factores de Williams y Thorn (1989) la proporción de varianza explicada se redujo a un $47 \%$. Por este motivo se optó por una solución de cuatro dimensiones: Dolor como un Misterio, Autoinculpación, Constancia, y Permanencia. Los coeficientes de consistencia presentados para las dimensiones fueron todos adecuados $(\alpha \geq .80)$.

En la versión italiana del PBPI (Monticone et al., 2013), el instrumento se adaptó a una muestra de 167 pacientes. Los autores lograron replicar la estructura original de tres factores, que en conjunto explicaban un $80 \%$ de la varianza; dimensiones denominadas Tiempo, Misterio, y Autoinculpación. Inversamente a lo observado en la versión inglesa (Morley \& Wilkinson, 1995), al forzar una estructura factorial de cuatro factores sólo se logra explicar un $40 \%$ de la varianza. Por este motivo se optó por la solución de tres factores. Asimismo, todas las dimensiones obtuvieron adecuados coeficientes de confiabilidad $(\alpha>$.90).

En la versión española del instrumento, Gonzales et al. (2002) hicieron uso del análisis factorial confirmatorio con el objetivo de validar la estructura de cuatro factores (i.e. Autoinculpación; Misterio; Constancia; y Temporalidad), en una muestra de 353 pacientes con dolor crónico y agudo. Para este fin, se compararon los índices de ajustes obtenidos para un modelo de cuatro y tres factores. El modelo de cuatro factores fue el que mostró mejores índices de ajustes $\left(\mathrm{X}^{2}=186.72 ; \mathrm{GFI}=.97\right.$; $\left.\mathrm{RMSEA}=.05 ; \mathrm{TLI}=.95 ; \mathrm{CFI}=.97\right)$.

En última instancia, recientemente se ha adaptado una versión rusa del inventario en una muestra de 250 pacientes con dolor crónico heterogéneo (Goli et al., 2015). En el estudio se verificó la estructura factorial de cuatro factores que explicaban un $62.7 \%$ del total de la varianza: 
Misterio, Permanencia, Constancia, y Autoinculpación. Los coeficientes de consistencia interna alcanzados por las dimensiones fueron aceptables $(\alpha>$.73).

Como puede apreciarse a partir de la revisión de los estudios antecedente no existe acuerdo entre los investigadores en relación a la estructura interna del instrumento. Concretamente, se observa divergencias en relación a la cantidad de factores subyacentes, así como de su interpretación. Mientras algunos investigadores obtienen tres factores (Monticone et al., 2013; Williams \& Thorn, 1989) otros reportan cuatro (Dany et al., 2009; Goli et al., 2015). Sumado a ello, la interpretación que se realiza de estos factores no es homogénea dado que algunas dimensiones (e.g., aceptación) no se replican en los restantes trabajos. Finalmente se aprecia que los ítems presentan una elevada variabilidad en relación a la saturación factorial ya que cargan en uno u otro factor dependiendo del estudio contemplado.

Un aspecto a considerar que podría estar influyendo en el comportamiento de los reactivos, y en consecuencia afectando sus propiedades métricas, es que los antecedentes revisados no contemplan la existencia de diferentes sub-grupos dentro de la población general de personas con dolor crónico. Tal como señalan los estándares psicométricos internacionales (AERA; APA; NCME, 2014), en determinados contextos es esperable que las propiedades psicométricas varíen entre subgrupos. De esta manera un mismo reactivo (e.g. "soy la causa de mi dolor" o "Mi dolor va a permanecer") puede presentar un funcionamiento muy distinto según el tipo de dolor crónico considerado (e.g. fibromialgia o cefalea). En función de ello la literatura psicométrica internacional recomienda analizar las propiedades psicométricas de una prueba atendiendo a las especificidades de cada sub-grupo cuando exista evidencia que sugiera que el constructo se comporta de manera diferencial. Este aspecto que no ha sido contemplado en los estudios antecedentes, incluyendo en sus muestras de estudios padecimientos crónicos diferentes.

Debido a la importancia que posee el contar con instrumentos que permitan medir variables predictoras (Oliveira, Dahlquist, Pinder \& Linhares, 2017) de la intensidad del dolor, como son las percepciones y creencias ante éste, y tomando esto en consideración las sugerencias de la literatura psicométrica internacional (AERA; APA; NCME, 2014), el presente trabajo tuvo por objetivo evaluar las propiedades psicométricas del PBPI en la población argentina, contemplando a personas con un mismo padecimiento crónico. Más concretamente se optó por trabajar con cefaleas crónicas, por tratase de un padecimiento de alta prevalencia (Visens, 2014).

\section{Participantes}

\section{Metodología}

Mediante un muestreo no probabilístico intencional se seleccionaron 382 personas de la ciudad de Córdoba (Argentina) que en los últimos seis meses habían sufrido de cefaleas. La muestra estuvo compuesta por un $77,7 \%$ de mujeres y un $22,3 \%$ de varones con una media de edad de 22.4 años (SD= 4.2). Es preciso destacar que el tamaño de la muestra cumple con los criterios estipulados por Hogan (2004) de contar al menos con cinco participantes por ítem.

\section{Instrumentos}

Percepciones y Creencias ante el Dolor. Para la presente investigación se partió de la versión adaptada del Inventario de Percepciones y Creencias ante el Dolor (IPCD, en adelante) González et al. (2002). El instrumento consta de dieciséis ítems que miden distintas percepciones y creencias hacia el dolor, que refieren originalmente a la autoinculpación ("Soy la causa de mi dolor"), la percepción del dolor como un misterio ("Nadie ha sido capaz de decirme exactamente por qué tengo este dolor") y las creencias acerca de la duración del dolor ("Mi dolor va a permanecer"). Las opciones de respuestas utilizadas en el presente estudio siguieron un formato tipo Likert con 5 categorías, desde totalmente en desacuerdo hasta totalmente de acuerdo. 
Cefaleas. Con el fin de evaluar los episodios de dolor de cabeza se construyó un cuestionario ad hoc donde los participantes informaban si habían sufrido algún episodio de cefalea en los últimos seis meses y su recurrencia. En el caso en que respondieran afirmativamente a este ítem, se les pedía que indicaran la frecuencia de aparición de los episodios de dolor de cabeza en una escala Likert de cinco puntos desde pocas veces al año a cada día de la semana.

Intensidad de Dolor. Para medir esta variable se empleó la Escala Visual Análoga, la cual es considerada un instrumento sensible a los cambios observados en las medidas que otras escalas para medir intensidad de dolor (Miró, 2006; Torres \& Compañ, 2006). Dicha escala mide de manera unidimensional la intensidad de dolor percibida por los pacientes. Esta escala consiste en una línea de 10 centímetros de longitud que va del cero (ningún dolor) a diez (el peor dolor imaginable) a lo largo de la cual los sujetos deben realizar una marca que represente la intensidad de dolor percibida (Chapman \& Syrjala, 2001). Este instrumento, además de ser uno de los más utilizados en la evaluación unidimensional del dolor (Torres \& Compañ, 2006), no necesita de adaptación psicométrica ya que para realizar estudios de consistencia interna y estructura interna se debe contar con un grupo de ítems o variables (Thompson, 2004), y en este caso se trata de un solo reactivo.

Ahora bien, con el fin de comenzar con el proceso de validación, se realizó una prueba piloto del IPCD con el objetivo de evaluar el funcionamiento del instrumento para poder detectar deficiencias y, de este modo, poder corregirlas.

\section{Procedimiento}

El presente estudio utilizó un diseño de tipo instrumental (Montero \& León, 2007), a partir del cual se pretende realizar la validación del IPCD. Una vez realizada la prueba piloto del instrumento, se llevó a cabo la administración de la escala por colaboradores debidamente entrenados. Es preciso mencionar que antes de comenzar con la investigación, los participantes fueron informados acerca de los fines de la investigación y del carácter anónimo y confidencial de sus respuestas. Todos los examinados optaron por participar de manera voluntaria, y aquellos que negaran su participación no recibieron consecuencias negativas por su decisión.

Respecto al análisis de datos, inicialmente se calcularon estadísticos descriptivos (e.g. media, desviación estándar, asimetría y curtosis) para conocer el comportamiento de las variables y el cumplimiento de los supuestos estadísticos. Tomando en consideración la ausencia de un modelo teórico de base claramente delimitado, ya que las evidencias reportadas por los estudios antecedentes son discordantes entre sí, se optó por utilizar un abordaje de carácter exploratorio para analizar la estructura interna del instrumento. Más aun considerando que no se cuenta con estudios previos que hayan indagado la estructura factorial del PBPI específicamente en personas con cefaleas crónicas.

Para el análisis factorial exploratorio se seguirán las recomendaciones brindadas por Ferrando y Lorenzo-Seva (2014) y Lloret-Segura, Ferreres-Traver, Hernández-Baeza y TomásMarco (2014). En el presente trabajo se considerará, a) la matriz de correlaciones productomomento de Pearson; b) la medida de Kaiser-Meyer-Olkin (KMO), y la prueba de esfericidad de Bartlett, para verificar la adecuación de los datos; c) la regla de Kaiser de autovalores mayores a uno, y el gráfico de sedimentación, para la determinación del número de factores; d) el método de estimación de Máxima Verosimilitud; y e) el procedimiento de rotación oblicua Promax, que contempla la posibilidad de que los factores a estimar correlacionen entre sí. La consistencia interna se evaluó utilizando el coeficiente Alfa de Cronbach. Finalmente, se utilizó el coeficiente de correlación de Pearson para analizar si existía una relación significativa entre los factores identificados y los puntajes de intensidad del dolor, analizando así si el instrumento cuenta con evidencias de validez de criterio. Todos los análisis fueron llevados a cabo por medio del software estadístico IBM SPSS 20. 


\section{Prueba piloto}

\section{Resultados}

Para la prueba piloto se reclutaron de manera accidental a doce personas con dolor crónico (seis mujeres y seis varones), y se entrevistó a los participantes para evaluar diferentes aspectos vinculados al funcionamiento de la prueba. En primera instancia se analizó la familiaridad con el vocabulario y expresiones utilizadas en los ítems y en las instrucciones, el tiempo que insumía la administración, la motivación de los participantes para responder, la adecuación del formato de codificación de las respuestas, y la comprensión de los ítems e instrucciones. También se analizó si las preguntas o las instrucciones suscitaban actitudes ego defensivas, es decir, que si los examinados sentían menoscabado su orgullo o autoestima por las preguntas de la prueba. Tal como recomienda Grasso (2006), al indagar sobre el detrimento que genera una determinada sintomatología sobre la salud mental o equilibrio emocional, pueden generarse actitudes defensivas en los examinados y afectar la sinceridad de sus respuestas.

Finalmente, y siguiendo las recomendaciones de la APA (2014), se evaluó el proceso cognitivo implicado en las respuestas de los participantes. Para ello se solicitó a los participantes que expresaran verbalmente sus pensamientos al momento de responder cada ítem, y se analizó la congruencia entre el constructo medido y la naturaleza de las respuestas emitida por los examinados.

Los participantes realizaron sugerencias respecto del instrumento administrado, a partir de las cueles se modificaron algunas palabras y expresiones poco claras no utilizadas con frecuencia en el lenguaje coloquial. También se cambiaron algunas expresiones de las instrucciones de los cuestionarios con el fin de mejorar su comprensión, y, finalmente, algunos ítems que estaban redactados en negativo se volvieron a redactar para optimizar su entendimiento.

\section{Evidencias de Estructura Interna y análisis de Consistencia Interna}

Se verificó en primera instancia la adecuación de la matriz de correlación para efectuar el análisis factorial exploratorio. Para esto, se consideró el test KMO, y el test de esfericidad de Bartlett (LloretSegura, et al., 2014). Los resultados del análisis inicial dan cuenta de la adecuación de los datos, tanto en la prueba de Bartlett $\left(\chi^{2}=1758,798 ; g l=120 ; \mathrm{p}<, 001\right)$ como en la medida KMO (KMO = ,82).

Al haber verificado la adecuación de los datos, se llevó a cabo el análisis factorial exploratorio. En este primer análisis se presentaron cuatro factores con autovalores superiores a uno, a la vez que el gráfico de sedimentación (screeplot) también sugiere la extracción de cuatro dimensiones, las cuales explican $58,63 \%$ de la varianza. A continuación, se muestran las cargas factoriales luego de la rotación Promax, correspondientes a los factores extraídos (tabla 1). 
Tabla 1

Matriz de Estructura. Extracción de cuatro factores. Rotación Promax. Las cargas menores a 30 han sido suprimidas.

\begin{tabular}{|c|c|c|c|c|}
\hline Ítems & Factor 1 & Factor 2 & Factor 3 & Factor 4 \\
\hline $\begin{array}{l}\text { Nadie ha sido capaz de decirme } \\
\text { exactamente por qué tengo este dolor }\end{array}$ & .726 & & & \\
\hline $\begin{array}{l}\text { Pensaba que mi dolor tenía curación, pero } \\
\text { ahora no estoy tan seguro/a }\end{array}$ & .629 & .430 & & -.377 \\
\hline En ocasiones no tengo ningún dolor & & -.300 & & \\
\hline Mi dolor me resulta confuso & 690 & .346 & & \\
\hline Mi dolor va a permanecer & .491 & .585 & & -.389 \\
\hline Continuamente tengo dolor & .383 & .774 & & \\
\hline Si tengo dolor es por mi culpa & & & ,819 & \\
\hline No sé lo suficiente acerca de mi dolor & .667 & & & \\
\hline $\begin{array}{l}\text { El dolor es un problema pasajero en mi } \\
\text { vida }\end{array}$ & & -.444 & & .417 \\
\hline $\begin{array}{l}\text { Es como si me levantara con el dolor y me } \\
\text { acostara con el dolor }\end{array}$ & .361 & .713 & & \\
\hline Soy la causa de mi dolor & & &, 855 & \\
\hline Hay una curación para mi dolor & & & & .380 \\
\hline Me culpo a mí mismo/a si tengo dolor & & & ,709 & \\
\hline $\begin{array}{l}\text { No puedo imaginarme por qué tengo este } \\
\text { dolor }\end{array}$ & .588 & & & \\
\hline $\begin{array}{l}\text { Algún día estaré completamente libre del } \\
\text { dolor }\end{array}$ & & & & .498 \\
\hline $\begin{array}{l}\text { Mi dolor varía en intensidad, pero está } \\
\text { siempre conmigo }\end{array}$ & .401 & .792 & & \\
\hline
\end{tabular}

Las correlaciones obtenidas entre los factores se muestran a continuación (tabla 2).

Tabla 2

Matriz de correlación de factores.

\begin{tabular}{lcccc}
\hline & Factor 1 & Factor 2 & Factor 3 & Factor 4 \\
\hline Factor 1 & - & .483 & .244 & -.223 \\
Factor 2 & & - & .149 & -.432 \\
Factor 3 & & & - & .102 \\
Factor 4 & & & & - \\
\hline
\end{tabular}

Con el objetivo de obtener una estructura factorial más simple e interpretable teóricamente, se procedió a repetir los análisis, pero en este caso extrayendo tres factores. Mediante esta estructura trifactorial se explica el 51,46\% de la varianza. A continuación, se describen las cargas obtenidas para cada factor (tabla 3 ). 
Tabla 3

Matriz de Estructura. Extracción de 3 factores. Rotación Promax. Las cargas menores a .30 han sido suprimidas.

\begin{tabular}{lccc}
\hline Ítems & Factor & Factor & Factor \\
& 1 & 2 & 3 \\
\hline Nadie ha sido capaz de decirme exactamente por qué tengo este dolor & .722 & & \\
Pensaba que mi dolor tenía curación, pero ahora no estoy tan seguro/a & .626 & .424 \\
Mi dolor me resulta confuso & .690 & \\
Mi dolor va a permanecer & .506 & .581 \\
Continuamente tengo dolor & .406 & .764 \\
Si tengo dolor es por mi culpa & & & \\
No sé lo suficiente acerca de mi dolor & .663 & & \\
Es como si me levantara con el dolor y me acostara con el dolor & & .711 \\
Soy la causa de mi dolor & & & \\
Hay una curación para mi dolor & & & \\
Me culpo a mi mismo/a si tengo dolor & & & \\
No puedo imaginarme por qué tengo este dolor & & & \\
Algún día estaré completamente libre del dolor & .588 & & \\
Mi dolor varía en intensidad, pero está siempre conmigo & .784 & \\
\hline
\end{tabular}

Los valores obtenidos permiten observar la presencia ítems con cargas muy débiles en las dimensiones extraídas (menores a ,30). En función de esto, se procedió a eliminar aquellos ítems con cargas débiles; esto es, ítems: 12 ("Hay una curación para mi dolor"); 15 ("Algún día estaré completamente libre del dolor"), 9 ("El dolor es pasajero en mi vida) y 3 ("En ocasiones no tengo ningún dolor"). Por último, si bien algunos ítems presentaron cargas complejas, se decidió mantenerlos dado que la carga más fuerte se encontró siempre en el factor teóricamente correspondiente, y en función de la coherencia que la carga factorial mantenía con los restantes ítems.

Las correlaciones obtenidas entre los factores se exponen a continuación (tabla 4). 
Tabla 4.

Matriz de correlación de factores.

\begin{tabular}{lccc} 
& Factor 1 (Dolor como un & Factor 2 (Cronicidad del & $\begin{array}{c}\text { Factor 3 } \\
\text { (Autoinculpación) }\end{array}$ \\
\hline Factor 1 & - & Dolor) & .267 \\
Factor 2 & & .490 & .169 \\
Factor 3 & & - & - \\
\hline
\end{tabular}

Se analizaron posteriormente los coeficientes de consistencia interna de los tres factores obtenidos. Los tres factores obtuvieron valores óptimos: Dolor como un Misterio $(\alpha=, 79)$, Autoinculpación $(\alpha=, 83)$ y Cronicidad del Dolor $(\alpha=, 83)$.

\section{Evidencias de Validez de Criterio}

Con el fin de obtener evidencia de validez de criterio, se procedió a verificar la relación entre los factores obtenidos (i.e. Dolor como un Misterio; Autoinculpación; y Cronicidad del Dolor) y la Intensidad del Dolor. Pudo observarse que la Intensidad del Dolor muestra una relación positiva y baja con el Dolor como un Misterio $(\mathrm{r}=.24 ; \mathrm{p}<.001)$; y una relación positiva y moderada con la Cronicidad del Dolor $(\mathrm{r}=.37 ; \mathrm{p}<.001)$.

\section{Discusión}

En la actualidad se considera que el sistema de creencias que utiliza el paciente con dolor es un determinante de las estrategias que utilice para manejarlo y de la respuesta que éste tenga al tratamiento (La Roche, Adames \& Chavez-Dueñas, 2017) Por este motivo, una evaluación de las variables cognitivas asociadas al dolor debe incluir las creencias que posee el paciente acerca de su problema de dolor (Goli et al., 2015), por lo que contar con instrumentos adecuados para medir las creencias sobre el dolor resulta muy importante (Baird \& Haslam, 2013; Goli et al., 2015; Gonzales et al., 2002; Wong et al., 2011).

El presente trabajo se propuso adaptar el Inventario de Percepciones y Creencias ante el Dolor (PBPI). Como objetivo adicional se analizó el comportamiento psicométrico de la prueba en personas con cefaleas, es decir, un subgrupo especifico con padecimiento crónico. Tal como sugiere la normativa psicométrica internacional (APA, 2014) resulta relevante realizar estudios que contemplen a un subgrupo específico cuando exista evidencia que sugiera que las propiedades de la prueba y la interpretación de los puntajes presenten particularidades en relación al grupo general. En este caso se trabajó con personas con cefaleas, pero en futuras investigaciones deberían analizarse subgrupos con otros padecimientos crónicos (e.g., personas con fibromialgia).

Los resultados obtenidos en este trabajo son coherentes con el modelo de tres factores propuesto por Williams y Thorn (1989), replicado posteriormente por Monticone et al. (2013). Similar a dichos autores, en el presente trabajo se constataron 3 dimensiones con buenos índices de consistencia interna, referidas a las creencias de: Autoinculpación (e.g. "Continuamente tengo dolor"; $\alpha=, 83$ ); Dolor como un Misterio (e.g. "No sé lo suficiente acerca de mi dolor"; $\alpha=, 79$ ); y Cronicidad del Dolor (e.g. "Si tengo dolor es por mi culpa"; $\alpha=, 80$ ). Al igual que Monticone et al. (2013) y, a diferencia de lo observado en gran parte de los estudios precedentes (Condello et al., 2014; Dany et al., 2009; Goli et al., 2015; Gonzales et al., 2002; Herda et al., 1994; Mikail et al., 1995; Morley \& Wilkinson, 1995; Wong et al., 2011), no se obtuvo una solución aceptable al extraer cuatro factores. Sin embargo, cabe mencionar que en la presente investigación no todos los ítems saturaron en los factores correspondientes, y otros tuvieron que eliminarse dado que afectaban la consistencia interna de los constructos.

Por otra parte, la correlación observada entre los factores obtenidos ha sido similar a lo evidenciado en otros estudios (Goli et al., 2015; Gonzales et al., 2002; Wong et al., 2011). De este modo, pudo verificarse que la dimensión Cronicidad del Dolor correlaciona de manera alta y positiva con el factor Dolor como un Misterio, mientras que los factores Cronicidad del Dolor y Dolor como un Misterio correlacionan de manera baja y positiva con la Autoinculpación. Estos resultados son coherentes en términos teóricos y van en consonancia con los antecedentes empíricos. En efecto es esperable que las 
personas con dolores más crónicos tengan mayor incertidumbre en relación a como continuará su dolor y en consecuencia desarrollen creencias y pensamientos de desconcierto en relación al dolor. La íntima relación entre ambos factores se explica además por la existencia de cargas compartidas de ciertos ítems en ambos factores. A mayor cronicidad mayor incertidumbre en relación a los motivos que explican el inicio y mantenimiento del dolor.

Respecto a la validez de criterio, fue posible verificar que la Intensidad del Dolor se correlaciona de manera positiva, aunque baja con el Dolor como un Misterio; y de forma positiva y moderada con la Cronicidad del Dolor. Finalmente, la Intensidad del Dolor no se correlacionó con la dimensión Autoinculpación. Estos resultados son coherentes con los estudios de Dany et al. (2009), Goli et al. (2015), Gonzales et al. (2002), Monticone et al. (2013) y Wong et al. (2011), quienes han encontrado asociaciones. Tal como sugieren Osborn y Smith (2015) la forma en que las personas perciben su dolor, es decir el significado atribuido, así como la valoración que las personas hacen respecto de la permanencia del dolor, su origen y la negatividad atribuida determinan en gran medida la intensidad del dolor que perciben. Asimismo, podría conjeturarse que aquellas personas que sostienen el tipo de creencias ante el dolor ya mencionadas, tendrían un ajuste pobre al problema de dolor y a su tratamiento. En esta misma línea, Condello et al. (2015) encontraron que creencias disfuncionales asociadas a las cefaleas reportadas por los pacientes predecían un afrontamiento pobre ante el dolor en función de la frecuencia de los episodios cefaleicos, teniendo un impacto negativo sobre el ajuste al problema de dolor.

Es importante notar la relevancia de los hallazgos obtenidos y del hecho de contar con un instrumento válido y confiable que permita realizar una evaluación completa del problema de dolor de los pacientes, lo que impactaría directamente en la planificación estratégica del tratamiento a seguir. 


\section{Referencias}

American Educational Research Association, American Psychological Association, National Council on Measurement in Education (2014). Standards for educational and psychological testing. American Educational Research Association.

Baird, A.J. \& Haslam, R.A. (2013). Exploring Differences in Pain Beliefs Within and Between a Large Nonclinical (Workplace) Population and a Clinical (Chronic Low Back Pain) Population Using the Pain Beliefs Questionnaire. Physical Therapy, 93(12), 1615-1624. https://academic.oup.com/ptj/article/93/12/1615/2735292

Bylinka, J. \& Oniszczenko, W. (2016). Temperament, Beliefs About Pain Control, and Pain Intensity in Endometriosis Patients. Journal of Clinical Psychology in Medical Settings, 23(4), 410-419. https://link.springer.com/article/10.1007/s10880-016-9473-8

Camacho Martel, L. \& Anarte Ortiz, M. T (2001). Importancia de las creencias en la modulación del dolor crónico: Concepto y evaluación. Apuntes de Psicología, 19 (3), 453-470.

Chapman, C. R. \& Syrjala, K. L. (2001). Measurement of pain. En J. D. Loeser (Eds.). Bonica's Management of Pain. Philadelphia: Lippincott Williams \& Wilkins Publishers.

Condello, C.; Piano, V.: Dadam, D.; Pinessi, L. \& Lantéri-Minet, M. (2015). Pain beliefs and perceptions inventory: a cross-sectional study in chronic and episodic migraine. Headache, 55(1), 136-48. https://www.ncbi.nlm.nih.gov/pubmed/25545038

Dany, L., Roussel, P., Carayon, S., Blois, S., \& Apostolidis, T. (2009). French adaptation and validation of the pain beliefs and perceptions inventory (PBPI). Pratiquespsychologiques, 15, 387-404.

DeGood, D. E. \& Shutty, M. S. (1992). Assessment of pain beliefs, doping and self-efficacy. En D.C. Turk \& R. Melzack (Eds.) Handbook of pain assessment. New York: The Guilford Press.

Fergus, T.A. (2013). Health-related dysfunctional beliefs and health anxiety: Further evidence of cognitive $\begin{array}{llll}\text { specificity. Journal of Clinical Psychology, } & \text { 70(3), 248-259. }\end{array}$ https://onlinelibrary.wiley.com/doi/pdf/10.1002/jclp.22012

Ferrando, P.J. \& Lorenzo-Seva, U. (2014). El análisis factorial exploratorio de los ítems: algunas consideraciones adicionales. Anales de Psicología, 30(3), 1170-1175. http://www.redalyc.org/pdf/167/16731690032.pdf

García-Camargo, F. J., \& Acuña, L. (2016). Relación entre experimentar eventos vitales estresantes y su severidad. Interamerican Journal of Psychology, 50(3), 380-391.

Goli, Z., Yanchuk, V., \& Torkaman, Z. (2015). Cross-cultural adaptation and validation of the Russian Version of the Pain Beliefs and Perceptions Inventory (R-PBPI) in patients with chronic pain. Current Psychology, 34 (4), 772-780. http://link.springer.com/article/10.1007/s12144-014-9289-3

González, R., Soler, E., \& Ferrer, V. A. (2002). Inventario de percepción y creencias de dolor: Análisis factorial confirmatorio. Revista Iberoamericana de Diagnóstico y Evaluación Psicológica, 14 (2), $135-148$.

Grasso, L. (2006). Encuestas. Elementos para su diseño y análisis. Córdoba: Encuentro Grupo Editor.

Herda, C. A., Siegeris, K., \& Basler, H-D. (1994). The Pain Beliefs and Perceptions Inventory: Futher evidence for a 4-factore structure. Pain, 57, 85-90.

Ho, P. \& Johnson, M. (2013). Behaviours and beliefs about pain and treatment among Chinese immigrants and New Zealand Europeans. The New Zealand Medical Journal, 126(1370), 1-3.

Jackson, T.; Wang, Y. \& Fan, H. (2014). Associations Between Pain Appraisals and Pain outcomes: MetaAnalyses of Laboratory Pain and Chronic Pain Literatures. The Journal of Pain, 15 (6), 586-301. http://www.jpain.org/article/S1526-5900(14)00563-X/abstract

La Roche, M. J., Adames, H. Y., \& Chavez-Dueñas, N. Y. (2017). Seven Implicit Considerations to be Explicitly Addressed in Empirically Based Psychotherapies. Interamerican Journal of Psychology, 51(2), 226-238.

Lloret-Segura, S.; Ferreres-Traver, A.; Hernández-Baeza, A. \& Tomás-Marco, I. (2014). El análisis factorial exploratorio de los ítems: una guía práctica, revisada y actualizada. Anales de Psicología, 30(3), 1151-1169. http://www.redalyc.org/pdf/167/16731690031.pdf 
Mikail, S. F., D’Eon, J. L., \& De Gagné, T. A. (1996). Validation of the Pain Beliefs and Perceptions Inventory. Pain Research Management, 1 (1), 31-42. https://www.hindawi.com/journals/prm/1996/134982/abs/

Miró, J. (2006). Dolor crónico, procedimientos de evaluación e intervención psicológica. Bilbao: Desclée de Brouwer.

Montero, I. \& León, O.G. (2007). Guía para nombrar los estudios de investigación en Psicología. International Journal of Clinical and Health Psychology, 7, 847-862. http://www.aepc.es/ijchp/GNEIP07_es.pdf

Monticone, M., Ferrante, S., Ferrari, S., Foti, C., Mugnai, R., Pillastrini, P., Rocca, B., \& Vanti, C. (2014). The Italian version of the Pain Beliefs and Perceptions Inventory: Cross-cultural adaptation, factor analysis, reliability and validity. Quality of Life Research, 23 (6), 1789-1795. http://link.springer.com/article/10.1007/s11136-013-0621-4

Moretti, L. S. (2010). Variables cognitivas Implicadas en la Experiencia de Dolor Crónico. Revista $\begin{array}{lllll}\text { Argentina de Ciencias del Comportamiento, 21-29. } & \text { 2, }\end{array}$ https://revistas.unc.edu.ar/index.php/racc/article/view/5246

Morley, S., \& Wilkinson, L. (1995). The pain beliefs and perceptions inventory: A British replication. Pain, 61, 427-4

Oliveira, N.C.; Dahlquist, L.M.; Pinder, W.M. \& Linhares, M.B.M. (2017). Cross-cultural adaptation of distress assessment instrument in children undergoing painful procedures. Revista Interamericana de Psicologia, 51(3), 398-405.

Osborn, M. \& Smith, J. A. (2015). The personal experience of chronic benign lower back pain: An interpretative phenomenological analysis. British Journal of Pain, 9 (1), 65-83. http://onlinelibrary.wiley.com/doi/10.1111/j.2044-8287.1998.tb00556.x/abstract

Pons, T.; Shipton, E. \& Mulder, R. (2012). The Relationship between Beliefs about Pain and Functioning with Rheumatologic Conditions. Rehabilitation Research and Practice, 2012, 1-9. https://www.hindawi.com/journals/rerp/2012/206263/

Sharp, T. J. (2001). Chronic pain: A reformulation of the cognitive- behavioural model. Behaviour Research and Therapy, 39, 787-800.

Stroud, M. W.; Thorn, B. E.; Jensen, M. P. \& Boothby, J. L. (2000). The relation between pain beliefs, negative thoughts, and psychosocial functioning in chronic pain patients. Pain, 84, 347-352.

Thompson, B. (2004). Exploratory and confirmatory factor analysis. Washington: American Psychological Association.

Torres, M. \& Comapñ, V. (2006). La experiencia de dolor. Barcelona: Editorial UOC.

Turk, D. C. \& Okifuji, A. (2002). Psychological factors in chronic pain: Evolution and revolution. Journal of Consulting and Clinical Psychology, 70, 678-690.

Vanhaudenhuysead, A.; Gilletb, A.; Malaisea, N.; Salamuna, I.; Grosdentc, S.; Maquetc, D.; Nyssenbd, A.S. \& Faymonville, M.E. (2018). Psychological interventions influence patients' attitudes and beliefs about their chronic pain. Journal of Traditional and Complementary Medicine, 8(2), 296302. https://www.sciencedirect.com/science/article/pii/S2225411016302656

Visens, L. S. (2014). Actualización en la prevención y tratamiento de la migraña. Medicina, 74 (2), $147-$ 157.

Williams, D. A., \& Thorn, B. E. (1989). An empirical assessment of pain beliefs. Pain, 36 (3), 351-358.

Wong, W. S., Williams, D. A., Mak, K. H., \& Fielding, R. (2011). Assessing attitudes toward and beliefs about pain among chinese patients with chronic pain: Validity and reliability of the Chinese Version of the Pain Beliefs and Perceptions Inventory (ChPBPI). Journal of Pain and Symptom Management, 42 (2), 308-318. 
Young Casey, C.; Greenberg, M. A.; Nicassio, P. M.; Harpin, R. E. \& Hubbard, E. (2008). Transition from acute to chronic pain and disability: A model including cognitive, affective and trauma factors. Pain, 134, 69-79.

Received:02/06/2017 Accepted: 07/09/2019 